\title{
Workflow updates to maintain clinical services and reduce utilisation of personal protective equipment during the COVID-19 outbreak
}

Hong Kong Med J 2020;26:263-4

https://doi.org/10.12809/hkmj208478

To the Editor-In a general hospital, there are many clinical procedures involving aerosol-generating procedures (AGPs), such as bronchoscopy and other specific procedures involving airway care, that can induce the production of aerosols of various sizes, including droplet nuclei. ${ }^{1}$ According to the latest guidelines from the Centre for Health Protection of the Hong Kong SAR Government, the recommended personal protective equipment (PPE) for performing AGPs includes N95 respirator, eye protection, gown, gloves, and cap (optional). ${ }^{2}$ However, there was a severe and mounting disruption to the global supply of PPE amid the outbreak of coronavirus disease 2019 (COVID-19). In response, the Hospital Authority adjusted public hospital non-emergency services and non-essential services to focus manpower and resources. ${ }^{3}$ Reducing utilisation of PPE in various clinical services has become an issue of current concern at frontline and management levels. ${ }^{4,5}$

Physiotherapists often perform AGPs such as open suctioning of respiratory tract (including tracheostomy care). In the Physiotherapy Department of Tuen Mun Hospital, the workflow of chest physiotherapy service was reviewed and analysed to explore the possibility of AGPs being grouped and handled by a designated team of physiotherapists, and the service delivery process of chest physiotherapy was then re-designed. A designated AGP team was established in which a group of physiotherapists (8 in total, on rotation) solely delivering chest physiotherapy involving AGPs. The workload for the AGP team was centralised and managed with extended working hours to maximise the use of every N95 respirator. To facilitate the implementation of the new workflow and compliance of infection control measures, a patient care assistant was assigned to assist the physiotherapist in logistics and patient preparation prior to and after the treatment, to maximise work efficiency. Additional training was provided to the patient care assistant to enhance their competency in infection control measures. The patient care assistant also helped to ensure proper gowning procedures of the physiotherapist. The changes in workflow were well communicated and supported by all staff. The AGP team using the new workflow have been observed by the consultant microbiologist and the cluster infection control officer, and they have found that the new workflow fulfils the updated requirements of infection control. Since implementing the new workflow in February 2020, consumption of PPE in the Physiotherapy Department has decreased substantially. The usage of N95 respirators decreased from approximately 60 pieces to eight pieces daily ( $>80 \%$ reduction), resulting in saving $>1000$ N95 respirators per month. Most importantly, such administrative change of workflow neither sacrificed the clinical service provision nor the occupational safety in performing high-risk AGPs. In addition to chest physiotherapy, the above measures may also be applicable to other clinical procedures involving AGP such as elective endotracheal intubation, bronchoscopy, and upper airway endoscopy.

\section{Author contributions}

All authors contributed to the concept of the study, acquisition and analysis of the data, drafting of the manuscript, and critical revision of the manuscript for important intellectual content. All authors had full access to the data, contributed to the study, approved the final version for publication, and take responsibility for its accuracy and integrity.

\section{Conflicts of interest}

All authors have disclosed no conflicts of interest.

\section{Funding/support}

This letter received no specific grant from any funding agency in the public, commercial, or not-for-profit sectors.

${ }^{1}$ Ken YT Lee *, PhD

${ }^{1}$ Aggie WS Kwan, MPH

${ }^{2}$ TL Que, MB, BS, FHKAM (Pathology)

${ }^{1}$ Mandy MY Mak, MSc

Department of Physiotherapy, Tuen Mun Hospital, Hong Kong

${ }^{2}$ Department of Clinical Pathology, Tuen Mun Hospital, Hong Kong

* Corresponding author: physioken@yahoo.com.hk

\section{References}

1. World Health Organization. Infection prevention and control of epidemic- and pandemic-prone acute respiratory diseases in health care. Available from: https://www.who. int/csr/resources/publications/WHO_CD_EPR_2007_6/ en/. Accessed 19 Mar 2020.

2. Centre for Health Protection, Department of Health, Hong Kong SAR Government. Recommended personal protective equipment (PPE) in hospitals/clinics under 
serious/emergency response level coronavirus disease (COVID-19). Available from: https://www.chp.gov.hk/ files/pdf/recommended_ppe_for_nid_eng.pdf. Accessed 19 Mar 2020

3. Hong Kong SAR Government. HA adjusts service provision to focus on combatting epidemic. Available from: https://www.info.gov.hk/gia/general/202002/10/ P2020021000711.htm. Accessed 19 Mar 2020.
4. Hong Kong SAR Government. Protective gear supply ensured. Available from: https://www.news.gov.hk/eng/20 20/03/20200313/20200313_180244_445.html. Accessed 19 Mar 2020.

5. Wong DH, Tang EW, Njo A, et al. Risk stratification protocol to reduce consumption of personal protective equipment for emergency surgeries during COVID-19 pandemic. Hong Kong Med J 2020;26:252-4. 